\title{
Inner-valence Auger decay in hydrocarbon molecules
}

\author{
Guoke Zhao, ${ }^{1,2}$ Tsveta Miteva, ${ }^{2}$ and Nicolas Sisourat ${ }^{2, *}$ \\ ${ }^{1}$ State Key Lab of New Ceramics and Fine Processing, \\ School of Materials Science and Engineering, Tsinghua University, Beijing 100084, China \\ ${ }^{2}$ Sorbonne Université, CNRS, Laboratoire de Chimie Physique Matière et Rayonnement, UMR 7614, F-75005 Paris, France \\ (Dated: June 21, 2019) \\ We have theoretically studied the Auger effect after inner-valence ionization of several unsaturated \\ and saturated cyclic and linear hydrocarbon molecules. These prototype molecules were chosen \\ such that the effects of the different characteristics of aromaticity ( $\pi$ electrons, conjugation, cyclic \\ geometry) on the Auger decay can be investigated separately. We show that among these molecules, \\ the ones having $\pi$ electrons can undergo Auger decay after inner-valence ionization. Furthermore, \\ the results reported here suggest that conjugation allows for several open Auger decay channels \\ while aromaticity limits the range of the latter.
}

*nicolas.sisourat@upmc.fr 


\section{INTRODUCTION}

Auger effect is an autoionization mechanism in which an electron from an outer shell fills a vacancy created in an inner shell while the excess energy is used to emit a secondary (Auger) electron. This effect is generally associated with core-ionization since for most atoms and small molecules it is in general energetically forbidden for electronic states corresponding to ionization in valence shells. However, it was observed experimentally that Auger effect is possible after inner-valence ionization of aromatic molecules [1,2]. Despite the quite extensive experimental and theoretical works on single and double ionization of aromatic molecules (see [3-10] and [11] for a recent review on double ionization of conjugated and aromatic molecules), there is no theoretical study of inner-valence Auger effect in such molecules. Furthermore, what characteristics of aromaticity ( $\pi$ electrons, conjugation, cyclic geometry) enable the Auger effect is not known.

In the present work, we investigate inner-valence Auger effect in several hydrocarbon molecules: benzene which is the simplest example of an aromatic system; cyclohexane which is a "saturated benzene molecule" and thus presents no characteristics of aromaticity; all trans 1,3,5-hexatriene which exhibits a delocalized conjugated $\pi$ system as benzene but is non-cyclic and 1,5 hexadiene which has unconjugated $\pi$ electrons. By comparing the single- and doubleionization spectra of these four prototype molecules, we attempt to disentangle the effects of each characteristic on inner-valence Auger effect. It is expected that the molecular size does not play an important role in this comparative study since all these molecules are constituted by 6 carbon atoms.

It should be mentioned that inner-valence Auger effect is energetically open in alkali-metal atoms owing to the low binding energy of the outer-valence electron (see [12] and references therein). Inner-valence Auger effect was also observed in satellite states of neon cation [13] and in multiple inner-valence ionization of fluoro-methane [14].

The paper is organised as follows. In the next section we outline the computational approach used to compute the single- and double-ionization potentials (IP and DIP, respectively) and the Auger spectra of the molecules considered in this work. In Sec. III and IV, we present and discuss the IP and DIP of these molecules, respectively. In Sec. V, the results on inner-valence Auger decay are reported. The summary and conclusions are given in Sec. VI.

\section{METHODS AND COMPUTATIONAL DETAILS}

The ground state equilibrium geometries of the molecules were obtained by energy minimization at the MollerPlesset 2nd order (MP2) level using the MOLPRO package [15, 16]. The vertical single and double ionization spectra were computed with Algebraic Diagrammatic Construction (ADC) methods (see [17] for a recent review): The single ionization spectra were computed using the non-Dyson extended second-order ADC $(2) \mathrm{x}$ scheme for the one-particle Green's function $[18,19]$ while the two-particle $\mathrm{ADC}(2)$ method [20-22] was used to compute the double ionization spectra.

The Hartree-Fock energies, molecular orbitals and the integrals needed for the ADC calculations were computed with a split-valence triple-zeta basis set [23] augmented with one $d$ function using the GAMESS-US package [24]. Convergence of the results with respect to the basis set was checked.

In order to interpret the value of the DIP, we use a Koopmans' like formula for the DIP. The DIP corresponding to ionization from orbital $i$ and $j$ for triplet states is given by

$$
D I P^{T}=-\left(\epsilon_{i}+\epsilon_{j}\right)+[i i \mid j j]-[i j \mid i j] ; i \neq j
$$

and that for the singlet states are

$$
\begin{aligned}
& D I P^{S}=-\left(\epsilon_{i}+\epsilon_{j}\right)+[i i \mid j j]+[i j \mid i j] ; i \neq j \\
& D I P^{S}=-2 \epsilon_{i}+[i i \mid i i] ; i=j
\end{aligned}
$$

where $\epsilon_{i}$ is the $i^{\text {th }}$ Hartree-Fock orbital energy and $[i j \mid k l]$ are two-electron integrals in the chemist's notation. These formulas provide a simple interpretation of the double-ionization potential: the DIP is the energy required to remove one electron from orbital $i$ and another one from orbital $j\left(-\left(\epsilon_{i}+\epsilon_{j}\right)\right)$ plus the Coulombic repulsion energy between the two created positive charges $([i i \mid j j])$ and plus or minus the exchange energy $([i j \mid i j])$.

Using the IP and DIP, we have estimated the coincidence spectra between the kinetic energy of the photoelectron (reported in binding energy, BE) and that of the Auger electron (E). The coincidence spectra were computed as

$$
\sigma(B E, E)=\sum_{i=1}^{N_{I P}} \sum_{j=1}^{N_{D I P}} p_{i} \omega_{i, j} e^{-\alpha\left[\left(B E-I P_{i}\right)^{2}+\left(E-D I P_{j}\right)^{2}\right]}
$$


where $\omega_{i, j}$ is the weight for a given channel (decaying state $i$ and final state $j$ ), $p_{i}$ the spectroscopic amplitude, $N_{I P}$ and $N_{D I P}$ are the number of ADC singly- and doubly-ionized states, respectively. We assume that each peak has a Gaussian shape, with a full width at half maximum set to $0.5 \mathrm{eV}$, in order to approximately include vibrational broadening [3, 7]. The spectroscopic amplitudes are obtained from the $\operatorname{ADC}(2) \mathrm{x}$ calculations. Auger decay is expected to take place within a few femtoseconds [14] and should thus be the dominant deexcitation pathway. However, computing partial Auger rates (i.e. $\omega_{i, j}$ ) for these systems is a challenging task, beyond the scope of the present work. Here, they are approximated as follows: singlet final states generally contribute more to the Auger spectra than the triplet ones [3,25], the weight for a singlet state was thus set to three times that of a triplet one as in Ref. [3]. For a given spin symmetry all weights were set equal. Given these approximations, the relative intensity of the peaks may vary significantly from the exact one. As shown below, such qualitative description of the spectra is still useful to illustrate the effects of the different characteristics of the molecules on the Auger decay.

It should be noted that in the $\mathrm{ADC}(2)$ methods the absolute values of IP and DIP are expected to be accurate within about 1-2 eV. In order to correct for the inaccuracy of the calculations, it is a common practice to shift the IP and DIP spectra such that the lowest line in each spectrum matches the experimental value. This is not done here because the lowest DIP is not known accurately for all molecules considered in this study. Furthermore, the aim of this work is to compare the molecules between each other. The relative energies between the molecules on one hand and the difference in IP and DIP for each molecule on the other hand are obtained at the same order of perturbation theory and the same basis set quality. We therefore base our discussion on the unshifted spectra.

\section{SINGLE IONIZATION POTENTIALS}

The single-ionization spectra of benzene and cyclohexane are shown in Fig. 1 and those of all trans 1,3,5-hexatriene and 1,5-hexadiene are reported in Fig. 2. While we are primarily interested in the inner-valence region (above $20 \mathrm{eV}$ ), we first discuss the position of the lowest line in each spectrum. The reason is two-fold: first this value is accurately known for the four molecules which allows to estimate the accuracy of the calculations. Second, after Auger decay the molecules are in the lowest doubly-ionized states which correspond to ionization from the outermost orbitals. Comparison with the lowest lines of the single-ionization spectra may then help the interpretation of the DIP.

The lowest IPs obtained with $\mathrm{ADC}(2) \mathrm{x}$ are compared with available data for each molecule in Table I. The values differ by at most $9 \%$. Furthermore, the order of increasing IP is well reproduced: the lowest IP is that of hexatriene, which is significantly lower than that of benzene. One possible explanation for this difference is that after ionization, benzene loses its aromatic character since singly-ionized benzene does not follow the $(4 n+2) \pi$ electrons rule [26]. The stabilization resonance energy of benzene adds therefore to the ionization energy of a conjugated $\pi$ electron system. Comparing hexatriene and hexadiene, we see that conjugation leads to a significantly lower IP. Hexadiene and benzene have similar IP while the IP of cyclohexane is only slightly larger.
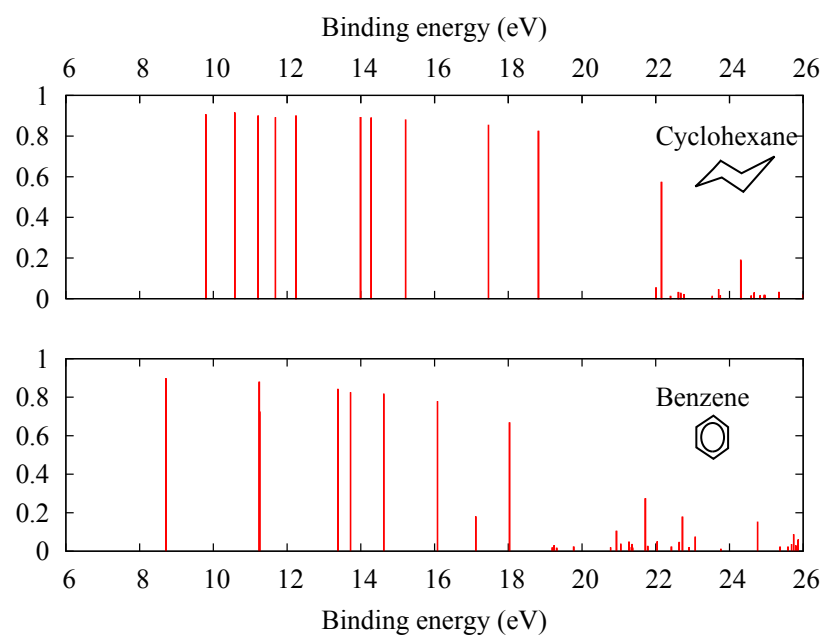

FIG. 1. Single ionization potentials of benzene and cyclohexane computed with ADC(2)x method.

We now turn the discussion to the inner-valence region. This energy range is characterized by the so-called breakdown of the molecular orbital picture [24]: because of the strong electronic correlation in this region, the singly-ionized states cannot be assigned as ionization mainly from a single molecular orbital. Instead electronic configurations cor- 

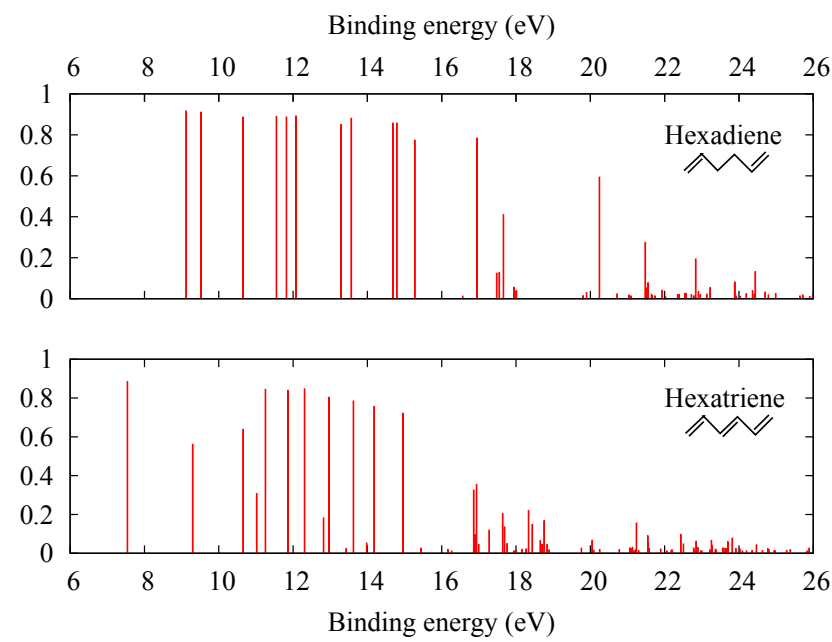

FIG. 2. Single ionization potentials of all trans 1,3,5-hexatriene and 1,5-hexadiene computed with the ADC(2)x method.

responding to ionization of one electron and excitation of another electron into an unoccupied orbital contribute significantly to each electronic state. Such configuration mixing is usually small in the outer-valence region and becomes strong in the inner-valence one which results in the presence of many lines with small spectroscopic amplitudes spread over several eVs.

Before comparing the spectrum of the molecules between each other, we first discuss the inner-valence region for the benzene molecule for which previous investigations are available. In [27] and [28], the ADC(3) method which provides a higher-order approximation of the spectrum was employed. At the ADC(3) level, the spectrum exhibits more peaks around $19 \mathrm{eV}$ than at the $\mathrm{ADC}(2)$ level. However, both methods predict inner-valence states with significant spectroscopic amplitudes up to $26 \mathrm{eV}$. As discussed below we are interested in states above the double ionization potential. The comparison between the two methods indicates that the $\mathrm{ADC}(2)$ method provides a sufficiently accurate spectrum for our study of inner-valence Auger decay.

The electronic states of hexatriene are the most strongly correlated ones since the breakdown of the molecular orbital picture appears already above $15 \mathrm{eV}$ while for the three other molecules the latter holds approximatively up to $20 \mathrm{eV}$. Furthermore, the spectrum of hexatriene exhibits a quite dense distribution of electronic states spreading from 20 to $25 \mathrm{eV}$. In contrast, for cyclohexane the inner-valence region is mostly characterized by only two fairly intense peaks which shows that the valence electrons of this molecule are weakly correlated. Above $20 \mathrm{eV}$, the spectra for all molecules having $\pi$ electrons are similar, exhibiting states with small spectroscopic amplitudes spread up to 26 eV.

Owing to the presence of numerous states with non-zero spectroscopic amplitudes in the inner-valence region for all molecules, the appearance of inner-valence Auger effect for a given molecule depends on the value of the lowest double ionization potential, i.e. a singly-ionized state can only undergo Auger decay if it lies above the double-ionization threshold.

\begin{tabular}{lcc}
\hline \hline & IP $(\mathrm{ADC}(2) \mathrm{x})$ & $\mathrm{IP}($ Expt. $)$ \\
\hline Benzene & 8.71 & $9.25^{a}$ \\
Cyclohexane & 9.79 & $9.88^{a}$ \\
Hexatriene & 7.54 & $8.30^{b}$ \\
Hexadiene & 9.12 & $9.27^{c}$ \\
\hline
\end{tabular}

TABLE I. Lowest single ionization potentials (in eV). ${ }^{a}$ Ref.[29], ${ }^{b}$ Ref.[30] and ${ }^{c}$ Ref.[31].

\section{DOUBLE IONIZATION POTENTIALS}

The double ionization spectra of benzene and cyclohexane are shown in Fig. 3 and those of all trans 1,3,5-hexatriene and 1,5-hexadiene are reported in Fig. 4. The lowest double ionization thresholds are given in Table II. 
The lowest double ionization threshold of benzene and cyclohexane corresponds to a triplet state characterized by ionization from the HOMO (i.e. highest occupied molecular orbital) and (HOMO-1). For hexatriene, owing to the low single ionization potential of the HOMO the lowest doubly-ionized state is a singlet state characterized by two holes in the HOMO. The lowest singlet and triplet states of hexadiene have nearly the same energy. In order to compare the four molecules we focus on the lowest triplet state. As seen in Table II, the latter is around $22-23 \mathrm{eV}$ for the three molecules having $\pi$ electrons while cyclohexane exhibits a much higher DIP lying about $2.5 \mathrm{eV}$ above the others. Such a significant difference may be expected from the single ionization potential of benzene and hexatriene compared to cyclohexane since the IPs of the former are about $1 \mathrm{eV}$ lower than that of the latter. Neglecting all correlation effects, one thus expects about $2 \mathrm{eV}$ difference between them. However, hexadiene and cyclohexane exhibit similar lowest IP. Using Koopmans' formulas (Eqs. 1-3) and the data given in Table III, it is seen that the difference between the DIPs of hexadiene and cyclohexane is due to the large exchange term for the former $(3.66 \mathrm{eV}$ instead of $0.26 \mathrm{eV})$.

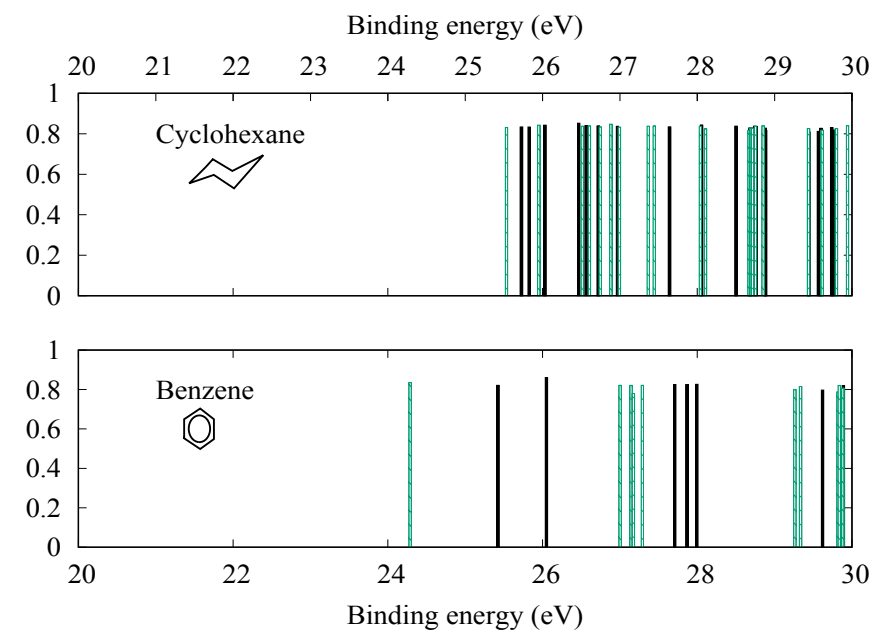

FIG. 3. Double ionization potentials of benzene and cyclohexane computed with ADC(2) method. Singlet and triplet states are shown in full black and dashed green bars, respectively.

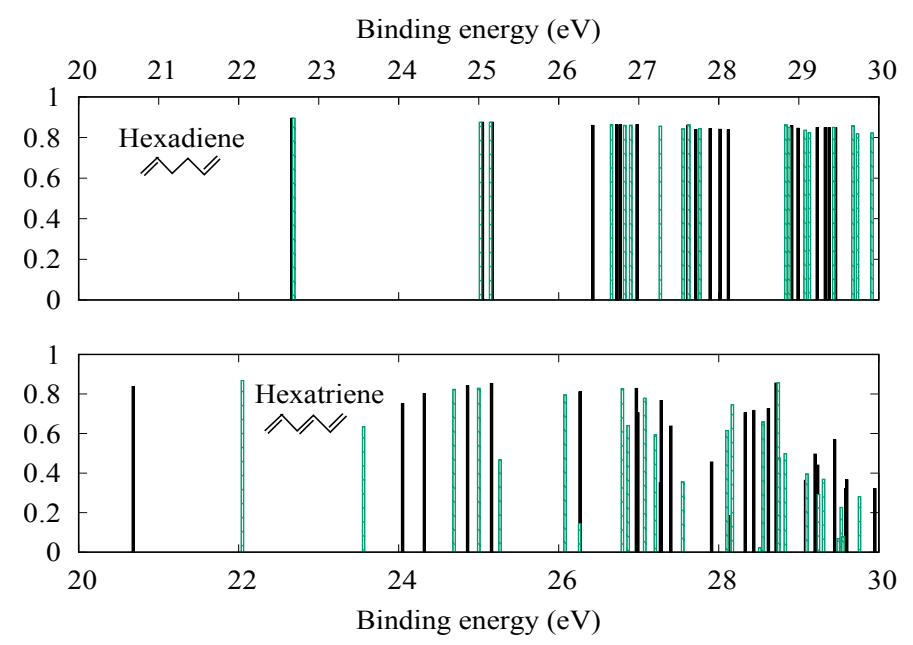

FIG. 4. Double ionization potentials of all trans 1,3,5-hexatriene and 1,5-hexadiene computed with ADC(2) method. Singlet and triplet states are shown in full black and dashed green bars, respectively.

We can use Koopmans' formulas to investigate further the behavior of the lowest DIP for the four molecules. From Table III, it is seen that the Coulombic repulsion energy between the charges is around $7 \mathrm{eV}$ for all molecules which indicates that neither $\pi$ electrons, nor conjugation or cyclic geometry have a significant contribution to the delocalization of the charges within the molecules. As shown in Eq. 1-3, the value of the DIP is dictated by the energy of the orbitals where the holes are created, the Coulomb energy and the exchange energy. Comparing benzene and hexatriene with hexadiene it is seen that conjugation lowers the energy of the orbitals. Furthermore, the comparison 
between the linear and the cyclic molecules shows that the exchange term is larger for the former which substantially lowers the lowest triplet state. Finally, the aromatic molecule exhibits the largest DIP among the molecules with $\pi$ electrons. Similarly to the case of single ionization, this may come from the loss of aromatic character after ionization.

\begin{tabular}{|c|c|c|c|c|}
\hline & \multirow{2}{*}{$\frac{\text { Triplet }}{1^{s t}}$} & Singlet & \multicolumn{2}{|c|}{ Expt. Triplet Expt. Singlet } \\
\hline & & $1^{\text {st }} \quad 2^{n d}$ & & \\
\hline Benzene & 24.25 & 25.4525 .91 & $24.65^{a}$ & - \\
\hline Cyclohexane & 25.53 & 25.7225 .82 & - & - \\
\hline Hexatriene & 22.04 & 20.6824 .04 & $24.3^{b}$ & $22.9^{b}$ \\
\hline Hexadiene & 22.69 & 22.6625 .04 & - & - \\
\hline
\end{tabular}

TABLE II. Lowest double ionization potentials (in eV) computed with $\mathrm{ADC}(2)$ method, compared to the experimental values when available: ${ }^{a}$ Ref. [2], ${ }^{b}$ Ref. [32]. Note that in [32], the authors employed Auger and double charge transfer spectroscopies to measure the DIP of several molecules. They have systematically discarded peaks observed at lower energies than the reported DIP. The latter might thus not be the lowest one for Hexatriene.

\begin{tabular}{llccccc}
\hline \hline & & $-\left(\epsilon_{i}+\epsilon_{j}\right)$ & {$[i i|| j j]$} & {$[i j \| i j]$} & $\mathrm{DIP}^{T}$ & $\mathrm{DIP}^{S}$ \\
\hline Benzene & $\mathrm{j}=\mathrm{N}-1$ & 18.56 & 7.31 & 0.60 & 25.26 & 26.47 \\
Benzene & $\mathrm{j}=\mathrm{N}$ & 18.56 & 8.53 & - & - & 27.09 \\
Cyclohexane & $\mathrm{j}=\mathrm{N}-1$ & 22.96 & 7.26 & 0.26 & 29.96 & 30.48 \\
Cyclohexane & $\mathrm{j}=\mathrm{N}$ & 22.96 & 7.79 & - & - & 30.75 \\
Hexatriene & $\mathrm{j}=\mathrm{N}-1$ & 18.72 & 6.63 & 2.26 & 23.09 & 27.61 \\
Hexatriene & $\mathrm{j}=\mathrm{N}$ & 15.88 & 7.06 & - & - & 22.94 \\
Hexadiene & $\mathrm{j}=\mathrm{N}-1$ & 19.97 & 7.22 & 3.66 & 23.53 & 30.85 \\
Hexadiene & $\mathrm{j}=\mathrm{N}$ & 19.54 & 7.49 & - & - & 27.03 \\
\hline
\end{tabular}

TABLE III. Hartree-Fock orbital energies and two-electron integrals used to compute Koopmans'like DIP (in eV). N is the number of occupied orbitals, e.g. $(\mathrm{i}=\mathrm{N} ; \mathrm{j}=\mathrm{N}-1)$ indicate that one electron from the HOMO and one from HOMO-1 are removed. Only data for $\mathrm{i}=\mathrm{N}$ are given.

\section{AUGER ELECTRON SPECTRA}

Comparing the single- and double-ionization spectra of each molecule, we first see that Auger decay after innervalence ionization is only possible for the unsaturated molecules. Indeed, for cyclohexane the lowest DIP is above all states of the singly-charged molecule having significant spectroscopic amplitudes. Given the fairly high IP for small saturated hydrocarbons it is expected that the double-ionization threshold is always higher than the inner-valence IP and therefore that inner-valence Auger decay is energetically forbidden for this kind of molecules.

For benzene, the singly-ionized electronic states in the highest part of the inner-valence region can undergo Auger decay. After the decay the lowest triplet state and the two lowest singlet states of benzene dications can be populated. An estimate of the kinetic energy of the Auger electron is given by the energy difference of the singly-ionized electronic states and the dicationic states. The energy distribution of the Auger electron after inner-valence ionization of benzene is thus between 0 and about $2.5 \mathrm{eV}$. Similar conclusions are drawn for hexadiene. For hexatriene, the doubly-ionized states are much lower in energy and more dicationic states can be populated by Auger decay. Furthermore, owing to the large distribution of singly-ionized states in the inner-valence region the kinetic energy distribution of the Auger electron spreads from 0 to about $5 \mathrm{eV}$.

The above discussion is illustrated by plotting the IP as a function of the kinetic energy of the Auger electron (see Eq. 4). It should be noted that such two-dimensional spectra can readily be measured with coincidence spectrometers 

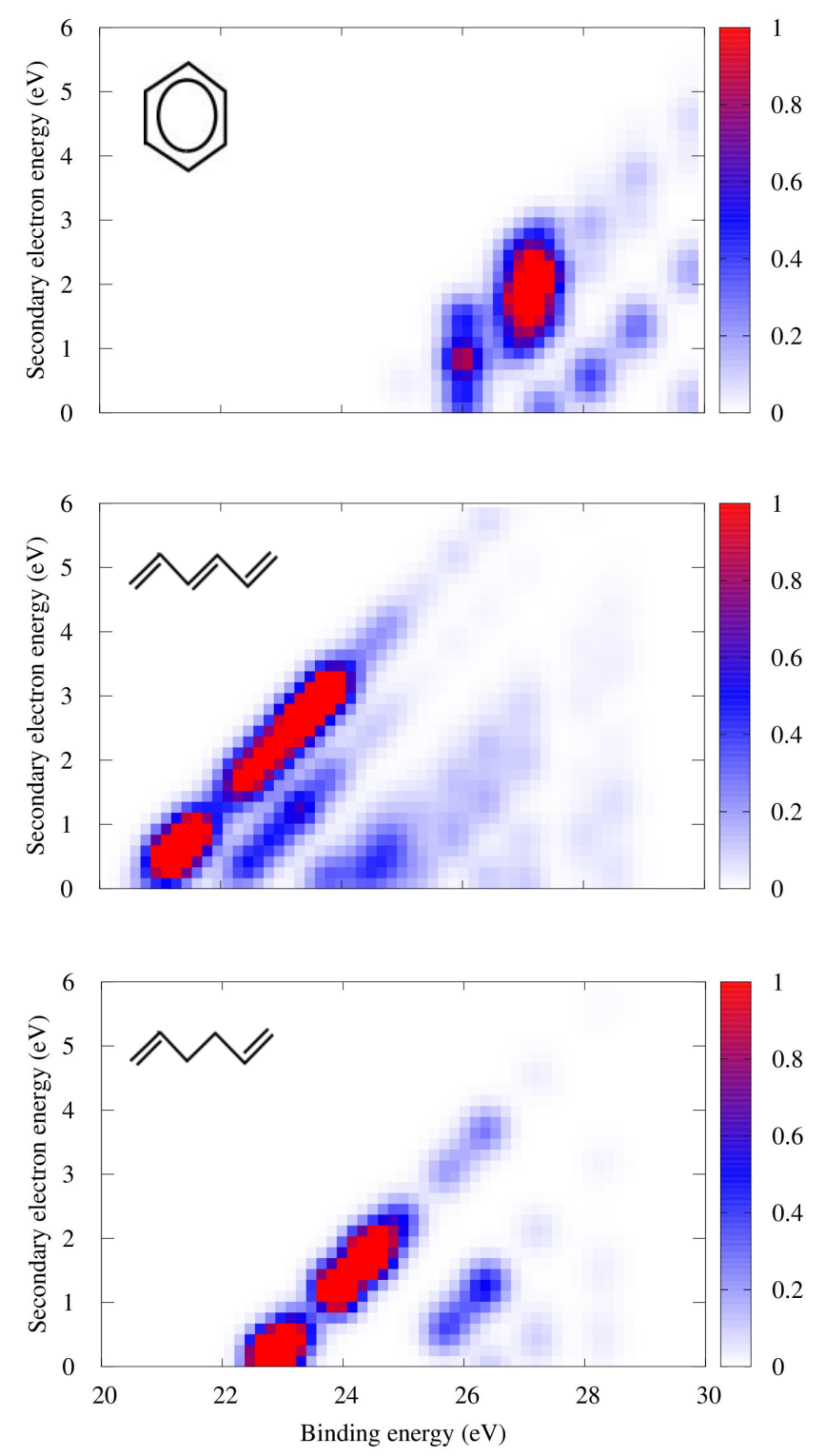

FIG. 5. Coincidence spectrum for benzene (upper-most panel), all-trans 1,3,5-hexatriene (middle panel) and 1,5-hexadiene (lower panel).

$[1,2,33,34]$. The coincidence spectra for benzene, all-trans 1,3,5-hexatriene and 1,5-hexadiene are shown in Fig. 5. The spectrum of benzene exhibits mainly two bands at around $(\mathrm{BE}=24 \mathrm{eV}, \mathrm{E}=1 \mathrm{eV})$ and $(\mathrm{BE}=26 \mathrm{eV}, \mathrm{E}=2 \mathrm{eV})$. The experimental coincidence spectrum for benzene is reported in [2]. Although the contribution of direct double photoionization (i.e. the simultaneous ejection of two photoelectron) is superimposed to the photoelectron - Auger electron spectrum, the features shown in the top panel of Fig. 5 are clearly seen in the experimental spectrum. For hexadiene, the coincidence spectrum exhibits a quasi-continuous diagonal line from $(\mathrm{BE}=23 \mathrm{eV}, \mathrm{E}=0 \mathrm{eV})$ to $(\mathrm{BE}=$ $25 \mathrm{eV}, \mathrm{E}=2 \mathrm{eV})$. As discussed above, the kinetic energy distribution of the Auger electron for hexatriene is broader than that for benzene and hexadiene. No experimental coincidence spectrum is available for these two molecules.

\section{CONCLUSIONS}

In conclusion, we have computed the single and double ionization spectra of benzene, cyclohexane, all-trans 1,3,5hexatriene and 1,5-hexadiene. These four molecules are prototypes of aromatic molecules, saturated compounds, 
non-aromatic conjugated and non-aromatic non-conjugated systems, respectively. We have investigated Auger decay after inner-valence ionization in these four molecules. Comparison between them provides insights into the effects of aromaticity, conjugation and $\pi$ electrons on the Auger decay. In particular, we have shown that the prototype molecules with $\pi$ electrons have a significantly lower double ionization threshold, which enables inner-valence Auger decay. The examples studied here indicate that conjugation leads to a larger number of open Auger decay channels. Within our approximations of the Auger intensities, conjugated molecules exhibit therefore broader kinetic energy distributions of the Auger electron. On the contrary, inner-valence Auger decay in the aromatic molecule is restricted to highly-lying inner-valence ionized states compared to the non-aromatic molecules, which may be explained by the loss of aromaticity of the former after single and double ionization. We hope that this work will encourage further theoretical and experimental investigations which are needed to evaluate the generality of the findings reported here.

\section{ACKNOWLEDGMENTS}

This project has received funding from the Research Executive Agency (REA) under the European Union's Horizon 2020 research and innovation programme Grant agreement No 705515 and from the LabEx MiChem part of French state funds managed by the ANR within the Investissements d'Avenir program under reference ANR-11-IDEX-000402 .

[1] R. D. Molloy and J. H. Eland, Chem. Phys. Lett. 421, 31 (2006).

[2] J. H. Eland, Chem. Phys. 345, 82 (2008).

[3] F. Tarantelli, A. Sgamellotti, L. S. Cederbaum, and J. Schirmer, J. Chem. Phys. 86, 2201 (1987).

[4] W. Griffiths, M. Langford, and F. Harris, Journal of the American Society for Mass Spectrometry 4, 513 (1993).

[5] P. Baltzer, L. Karlsson, B. Wannberg, G. Öhrwall, D. Holland, M. MacDonald, M. Hayes, and W. von Niessen, Chem. Phys. 224, 95 (1997).

[6] A. J. Yencha, R. I. Hall, L. Avaldi, G. Dawber, A. G. McConkey, M. A. MacDonald, and G. C. King, Can. J. Chem. 82, 1061 (2004).

[7] C. Villani and F. Tarantelli, J. Chem. Phys. 120, 1775 (2004).

[8] M. Schwell, H.-W. Jochims, H. Baumgärtel, and S. Leach, Chem. Phys. 353, 145 (2008).

[9] D. Ascenzi, J. Aysina, E.-L. Zins, D. Schroder, J. Zabka, C. Alcaraz, S. D. Price, and J. Roithova, Phys. Chem. Chem. Phys. 13, 18330 (2011).

[10] A. M. Śmiałek, A. M. MacDonald, S. Ptasińska, L. Zuin, and J. N. Mason, Eur. Phys. J. D 70, 1 (2016).

[11] J. Eland and R. Feifel, "Double photoionisation spectra of molecules," (Oxford University Press, 2017).

[12] J. Palaudoux, S. Sheinerman, J. Soronen, S.-M. Huttula, M. Huttula, K. Jänkälä, L. Andric, K. Ito, P. Lablanquie, F. Penent, J.-M. Bizau, S. Guilbaud, and D. Cubaynes, Phys. Rev. A 92, 012510 (2015).

[13] M. Kitajima, H. Yoshida, A. D. Fanis, G. Prmper, U. Hergenhahn, E. Kukk, T. Tanaka, K. Nakagawa, H. Tanaka, S. Fritzsche, I. P. Sazhina, N. M. Kabachnik, and K. Ueda, J. Phys. B 39, 1299 (2006).

[14] R. Feifel, J. H. D. Eland, R. J. Squibb, M. Mucke, S. Zagorodskikh, P. Linusson, F. Tarantelli, P. c. v. Kolorenč, and V. Averbukh, Phys. Rev. Lett. 116, 073001 (2016).

[15] H.-J. Werner, P. J. Knowles, G. Knizia, F. R. Manby, and M. Schütz, Wiley Interdisciplinary Reviews: Computational Molecular Science 2, 242 (2012).

[16] H.-J. Werner, P. J. Knowles, G. Knizia, F. R. Manby, M. Schütz, P. Celani, W. Györffy, D. Kats, T. Korona, R. Lindh, A. Mitrushenkov, G. Rauhut, K. R. Shamasundar, T. B. Adler, R. D. Amos, A. Bernhardsson, A. Berning, D. L. Cooper, M. J. O. Deegan, A. J. Dobbyn, F. Eckert, E. Goll, C. Hampel, A. Hesselmann, G. Hetzer, T. Hrenar, G. Jansen, C. Köppl, Y. Liu, A. W. Lloyd, R. A. Mata, A. J. May, S. J. McNicholas, W. Meyer, M. E. Mura, A. Nicklass, D. P. O’Neill, P. Palmieri, D. Peng, K. Pflüger, R. Pitzer, M. Reiher, T. Shiozaki, H. Stoll, A. J. Stone, R. Tarroni, T. Thorsteinsson, and M. Wang, "Molpro, version 2015.1, a package of ab initio programs," (2015).

[17] D. Danovich, Wiley Interdisciplinary Reviews: Computational Molecular Science 1, 377 (2011).

[18] J. Schirmer, A. B. Trofimov, and G. Stelter, J. Chem. Phys. 109, 4734 (1998).

[19] A. B. Trofimov and J. Schirmer, J. Chem. Phys. 123, 144115 (2005).

[20] J. Schirmer and A. Barth, Z. Phys. A 317, 267.

[21] F. Tarantelli, Chem. Phys. 329, 11 (2006), electron Correlation and Multimode Dynamics in Molecules(in honour of Lorenz S. Cederbaum).

[22] Y. Velkov, T. Miteva, N. Sisourat, and J. Schirmer, J. Chem. Phys. 135, 154113 (2011).

[23] R. Krishnan, J. S. Binkley, R. Seeger, and J. A. Pople, J. Chem. Phys. 72, 650 (1980).

[24] M. W. Schmidt, K. K. Baldridge, J. A. Boatz, S. T. Elbert, M. S. Gordon, J. H. Jensen, S. Koseki, N. Matsunaga, K. A. Nguyen, S. Su, T. L. Windus, M. Dupuis, and J. A. Montgomery, J. Comp. Chem. 14, 1347 (1993).

[25] H. Ågren, J. Chem. Phys. 75, 1267 (1981). 
[26] J. D. Roberts, A. S. Jr., and C. M. Regan, J. Am. Chem. Soc. 74, 4579 (1952).

[27] H.-G. Weikert and L. Cederbaum, Chem. Phys. Lett. 237, 1 (1995).

[28] V. Despré, A. Marciniak, V. Loriot, M. C. E. Galbraith, A. Rouze, M. J. J. Vrakking, F. Lépine, and A. I. Kuleff, J. Phys. Chem. Lett. 6, 426 (2015), pMID: 26261959.

[29] S. Lias, Ionization Energy Evaluation (NIST Chemistry WebBook, NIST Standard Reference Database Number 69, Eds. P.J. Linstrom and W.G. Mallard, National Institute of Standards and Technology, Gaithersburg MD, 20899, 2016).

[30] M. Allan, J. Dannacher, and J. P. Maier, J. Chem. Phys. 73, 3114 (1980).

[31] P. Wolkoffs, J. L. Holmes, and F. P. Lossing, Can. J. Chem. 58, 251 (1980), http://dx.doi.org/10.1139/v80-041.

[32] C.-M. Liegener, A. Naves de Brito, H. Ågren, N. Correia, W. J. Griffiths, S. Svensson, and F. M. Harris, Phys. Rev. B 46, 11295 (1992).

[33] F. Penent, J. Palaudoux, P. Lablanquie, L. Andric, R. Feifel, and J. H. D. Eland, Phys. Rev. Lett. 95, 083002 (2005).

[34] V. Ulrich, S. Barth, T. Lischke, S. Joshi, T. Arion, M. Mucke, M. Förstel, A. M. Bradshaw, and U. Hergenhahn, J. El. Spec. Rel. Phenom. 183, 70 (2011). 